\title{
ORIGINAL
}

\section{ANTROPOMETRIA DE ATLETAS CULTURISTAS EM RELAÇÃO À REFERÊNCIA POPULACIONAL}

\section{ANTHROPOMETRY OF BODY BUILDERS IN RELATION TO THE POPULATION STANDARD}

\author{
Naílza MAESTÁ' \\ Edilson Serpeloni CYRINO2 \\ Nelson Nardo JÚNIOR ${ }^{2}$ \\ Mônica Yara Gabriel MORELLI' \\ José Maria Santarém SOBRINHO ${ }^{3}$ \\ Roberto Carlos BURINI ${ }^{4}$
}

\begin{abstract}
RESUMO
Atletas de culturismo têm como alvo de treinamento a hipertrofia muscular e a redução da adiposidade. A falta de referências antropométricas apropriadas pode levar esses indivíduos a serem erroneamente considerados em situação de sobrepeso ou desnutrição. Portanto, objetivamos comparar a composição corporal de atletas de culturismo com os padrões populacionais. Foram avaliados 36 atletas, de ambos os sexos, 26 do sexo masculino (27,2 $\pm 7,2$ anos) e 10 do sexo feminino (30,0 $\pm 6,1$ anos), por ocasião da competição nacional, quanto aos indicadores antropométricos de peso, estatura, índice de massa corpórea, pregas cutâneas, adiposidade corpórea e circunferência muscular do braço. Os resultados foram referidos quanto à posição percentual ou de desvios-padrão (pelo escore Z) tendo como referências padrões populacionais locais. Como resultado, encontramos que os indicadores com menor contraste com os padrões adotados foram peso e estatura e os maiores contrastes foram encontrados na circunferência muscular do braço para os homens, e pregas cutâneas tricipital para as mulheres. Pelo indicador muscular todos foram classificados como obesos ou sobrepeso, enquanto que pela prega cutânea tricipital e adiposidade corpórea, a desnutrição esteve presente em 100,0\% das mulheres e 88,5\% dos homens. Fica evidenciada a inadequação da utilização do padrão antropométrico populacional para a classificação nutricional de atletas de força, em particular culturistas, sendo assim necessário o estabelecimento de padrões próprios para esse tipo de treinamento (modalidade).
\end{abstract}

Termos de indexação: estado nutricional, antropometria, atletas, musculação.

\footnotetext{
(1) Centro de Metabolismo e Nutrição (CeMeNutri), Faculdade de Medicina de Botucatu, Universidade Estadual Paulista.

(2) Departamento de Fundamento de Educação Física do Centro de Educação Física e Desportos (CEFD), Universidade Estadual de Londrina.

(3) Centro de Estudos em Ciências da Atividade Física (CECAFI), Disciplina de Geriatria, Faculdade de Medicina, Universidade de São Paulo.

(4) Departamento de Clínica Médica, Centro de Metabolismo e Nutrição (CeMeNutri), Faculdade de Medicina de Botucatu, Universidade Estadual Paulista. Caixa Postal 584 , 18618-970, Botucatu, SP, Brasil. Correspondência para/Correspondenceto: R.C. BURINI.
} 


\begin{abstract}
Body builders have as their training goals the maximum muscle hypertrophy with minimum adiposity. However, the scarcity of specific standards implies often in framing wrongly those athletes either as overweight (by their BMI) or energy malnourished (by their fat stores). The objective of this study was to compare the body composition of body builders with population standards. Thirty-six adults, 26 male $(27.2 \pm 7.2$ years $)$ and 10 female $(30 \pm 6.1$ years) nationwide competitive body builders, were assessed considering weight, height, body mass index, adiposity, arm and leg circumferences and skinfolds. The data were referred either as percentile or standard deviations (Z score) of population standards. Body weight and height were among the closest values from the populational mean whereas upper arm muscle circumference (for men) and body adiposity (for women) were the farterest. By using fat parameters as indicators of their protein-energy status, the undernourishment was found in $88.5 \%$ of men and $100.0 \%$ of women. Thus, it seems that body builders deserve their own anthropometric standards to avoid nutritional status misplacements.
\end{abstract}

Index terms: nutritional status, anthropometry, athletes, bodybuilders.

\section{INTRODUÇÃO}

O treinamento com pesos, forma mais comum de exercícios contra resistência ou resistidos, é considerado a atividade física mais eficiente para a modificação da composição corporal pelo aumento da massa muscular. Tais alterações na composição corpórea ocorrem após várias semanas de treinamento (Fleck \& Kraemer, 1997).

Atletas de culturismo (musculação de competição) apresentam porcentagens de gordura bastante abaixo dos padrões populacionais, apesar de ocorrer o inverso com a massa magra e até no peso corpóreo. Desta forma, esses atletas podem ser enquadrados, pelos padrões antropométricos populacionais, como desnutridos ou sobrepeso (World..., 1995), uma vez que ainda inexistem padrões (faixa normal) apropriados para esse tipo de população. Pretende-se pois, neste trabalho, levantar dados antropométricos comuns no estabelecimento da adiposidade e massa magra, em atletas culturistas representativos da elite nacional, e compará-los com os padrões existentes para não atletas.

\section{CASUÍSTICA E MÉTODOS}

\section{Casuística}

Foram avaliados 36 indivíduos, 26 do sexo masculino $(27,2 \pm 7,2$ anos) e 10 do sexo feminino $(30 \pm 6,1$ anos), todos atletas de culturismo em fase de competição, procedentes de diferentes regiões do país: Sudeste, $(37,2 \%)$, Nordeste $(32,6 \%)$ ou Sul $(18,6 \%)$.

\section{Métodos}

As medidas antropométricas, obtidas nas primeiras horas da manhã, foram compostas de peso e estatura, em balança antropométrica, da marca Filizola, para adultos, com precisão de 100 g, circunferência do braço, por meio de fita métrica inextensível com precisão de $0,1 \mathrm{~cm}$ e prega cutânea tricipital pela utilização de adipômetro (marca Cescorf), com pressão constante de $10 \mathrm{~g} / \mathrm{mm}^{2}$ e precisão de $1 \mathrm{~mm}$.

A partir desses dados foram calculados os valores de Índice de Massa Corpórea (IMC) $\left(\mathrm{kg} / \mathrm{m}^{2}\right)$ (Cronk \& Roche, 1982) e Circunferência Muscular do Braço (CMB) [Circunferência do Braço (CB) - (Prega Cutânea Tricipital $(\mathrm{PCT}) \times$ 0,314)] (Frisancho, 1981).

Para o cálculo da densidade corporal (D), foram utilizados dois métodos que se utilizam de Pregas Cutâneas: o de Petrosky (1995) para o sexo masculino, que leva em consideração as Pregas Subescapular (PCSE), Suprailíaca (PCSI), Tricipital (PCT) e Panturrilha (PCP), o de Pollock \& Jackson (1980) para o sexo feminino, que consideram as Pregas Abdominal (PCAb), Suprailíaca (PCSI), Tricipital (PCT) e Coxa (PCCX). Mediante a equação de Siri (1961), obteve-se a porcentagem de gordura corpórea (\%G), em ambos os sexos.

Os resultados foram utilizados para classificação do percentil (P) (Frisancho, 1981; Cronk \& Roche, 1982) e cálculo do escore Z (Sawaya, 1997) tendo o levantamento local (Anselmo et al., 1992) como padrão populacional, sendo a faixa normal $Z \pm 2 S D$.

\section{RESULTADOS E DISCUSSÃO}

Este estudo foi realizado durante um campeonato nacional, prévia para o mundial, portanto, com atletas de elite nacional em sua plena forma física. Os dados de estatura mostraram déficit (pelo escore Z) em 3 atletas masculinos e um valor acima do normal $(Z=3,7)$ dentre as atletas femininas. Os valores do peso corpóreo também foram bem próximos à distribuição populacional com exceção de um excesso de peso, dentre os atletas masculinos, classificados como sobrepeso II $(Z=2,37)$. Além desse atleta, outros dois, também do sexo masculino, apresentaram IMC compatível com obesidade. No geral, 
a média de IMC dos grupos foi de $27 \mathrm{~kg} / \mathrm{m}^{2}$ nos homens e $21 \mathrm{~kg} / \mathrm{m}^{2}$ nas mulheres, classificando o grupo masculino como sobrepeso I (Anselmo et al., 1992) (Tabela 1 e 2). Comparativamente aos dados da Tabela 3, verifica-se a semelhança dos resultados deste trabalho com os da literatura.

Pela análise das Tabelas 1 e 4 fica evidente que o sobrepeso masculino foi decorrente do excesso muscular pelos valores dos seus indicadores $\mathrm{CB}$ e $\mathrm{CMB}$ em contraposição aos valores reduzidos da PCT, aqui utilizado como indicador lipídico (Frisancho, 1981).

Como era esperado, o excesso de massa muscular foi mais freqüente no sexo masculino (Anselmo et al., 1992). A presença desse excesso foi detectada por ambos os indicadores ( $\mathrm{CB}$ e $\mathrm{CMB}$ ) no sexo masculino e apenas pela $\mathrm{CMB}(80,0 \%)$ no sexo feminino (Tabelas 1 e 4 e Figura 1$)$. A CB foi elevada $(Z=2,5)$ apenas em uma atleta feminina, exatamente a de maior valor de $\mathrm{CMB}$
$(Z=5,6)$ e menor valor de PCT $(Z=-3,42)$ (Tabelas 2 e 5). Os maiores desvios observados contra o padrão populacional foram os de $\mathrm{CMB}$, cujos valores de $\mathrm{Z}$ foram $3,5 \pm 1,4 \mathrm{~cm}$ e $6,8 \pm 1,8 \mathrm{~cm}$ respectivamente para os sexos feminino e masculino (Tabelas 1 e 2 e Figura 1 ).

Os padrões de referência para um homem de 70 $\mathrm{kg}$ (20-24 anos) com $170 \mathrm{~cm}$ de altura apresentam 15,0\% de gordura total, $45,0 \%$ de músculo e $15,0 \%$ de osso. $\mathrm{O}$ mínimo de gordura para um homem é em torno de 3,0\% (gordura constitucional essencial). O homem pesa mais que a mulher porque tem mais músculo, o esqueleto é mais pesado e ele é mais alto. Assim, a mulher se sobressai em relação ao homem quanto a quantidade de gordura corpórea, pois uma mulher de $163 \mathrm{~cm}$ de altura com $57 \mathrm{~kg}$ apresenta em torno de $27,0 \%$ de gordura total, 36,0\% de músculo e $12,0 \%$ de osso, só as mamas contribuem com $4,4 \%$ de gordura total. Além disso, existem depósitos gordurosos específicos do sexo feminino nas regiões

Tabela 1. Peso, altura, circunferência braquial e medidas derivadas de atletas culturistas do sexo masculino.

\begin{tabular}{|c|c|c|c|c|c|c|c|c|c|c|c|c|c|}
\hline & \multicolumn{2}{|c|}{ Peso } & \multicolumn{2}{|c|}{ Altura } & \multicolumn{3}{|c|}{ IMC } & \multicolumn{3}{|c|}{$\mathrm{CB}$} & \multicolumn{3}{|c|}{$\mathrm{CMB}$} \\
\hline & $\mathrm{kg}$ & Z & $\mathrm{cm}$ & Z & $\mathrm{kg} / \mathrm{m}^{2}$ & Z & Percentil & $\mathrm{cm}$ & Z & Percentil & $\mathrm{cm}$ & Z & Percentil \\
\hline 1 & 88,5 & 0,11 & 170 & $-0,34$ & 30,62 & 1,75 & $P_{90}$ & 43,5 & 3,80 & $>P_{95}$ & 40,7 & 6,50 & $>P_{95}$ \\
\hline 2 & 87,4 & 1,50 & 178 & 0,37 & 27,58 & 1,80 & $P_{90}$ & 43,0 & 5,40 & $>P_{95}$ & 40,4 & 6,10 & $>P_{95}$ \\
\hline 3 & 73,7 & $-0,02$ & 170 & $-0,34$ & 25,50 & 0,16 & $P_{50}$ & 41,0 & 3,50 & $>P_{95}$ & 39,7 & 6,00 & $>P_{95}$ \\
\hline 4 & 88,2 & 1,60 & 177 & 0,20 & 28,15 & 2,00 & $P_{75}$ & 43,2 & 4,70 & $>P_{95}$ & 40,4 & 9,40 & $>P_{95}$ \\
\hline 5 & 79,8 & 0,55 & 170 & $-0,34$ & 27,61 & 0,80 & $P_{75}$ & 40,1 & 3,30 & $>P_{95}$ & 37,8 & 5,20 & $>P_{95}$ \\
\hline 6 & 84,0 & 1,20 & 180 & 0,70 & 25,93 & 1,10 & $P_{75}$ & 40,1 & 3,30 & $>P_{95}$ & 36,9 & 7,30 & $>P_{95}$ \\
\hline 7 & 89,6 & 1,73 & 180 & 0,70 & 27,65 & 1,80 & $P_{75}$ & 47,5 & 3,30 & $>P_{95}$ & 44,6 & 12,0 & $>P_{95}$ \\
\hline 8 & 74,6 & 0,23 & 177 & 0,20 & 23,81 & 0,20 & $P_{50}$ & 39,0 & 2,80 & $>P_{95}$ & 36,0 & 6,70 & $>P_{95}$ \\
\hline 9 & 63,2 & $-0,99$ & 160 & $-2,10$ & 24,69 & $-0,10$ & $P_{50}$ & 40,2 & 2,80 & $>P_{95}$ & 37,5 & 5,00 & $>P_{95}$ \\
\hline 10 & 73,0 & $-0,08$ & 170 & $-0,34$ & 25,26 & 0,08 & $P_{50}$ & 40,5 & 2,90 & $>P_{95}$ & 38,1 & 5,30 & $>P_{95}$ \\
\hline 11 & 75,8 & 0,35 & 177 & 0,20 & 24,19 & 0,37 & $P_{75}$ & 40,5 & 2,90 & $>P_{95}$ & 37,8 & 7,80 & $>P_{95}$ \\
\hline 12 & 63,5 & $-0,38$ & 156 & $-3,30$ & 26,09 & 1,16 & $P_{75}$ & 39,2 & 3,30 & $>P_{95}$ & 37,4 & 7,60 & $>P_{95}$ \\
\hline 13 & 65,0 & $-0,73$ & 160 & $-2,63$ & 25,39 & 0,87 & $P_{75}$ & 37,0 & 2,30 & $<\mathrm{P}_{95}$ & 34,7 & 5,90 & $>P_{95}$ \\
\hline 14 & 96,0 & 2,37 & 170 & 0,37 & 30,30 & 2,90 & $P_{90}$ & - & & - & - & & \\
\hline 15 & 88,0 & 1,30 & 174 & 0,34 & 29,07 & 1,27 & $P_{90}$ & 45,0 & 4,80 & $>P_{95}$ & 43,8 & 7,90 & $>P_{95}$ \\
\hline 16 & 73,9 & 0 & 162 & 1,70 & 28,16 & 0,98 & $P_{75}$ & - & & - & - & - & - \\
\hline 17 & 72,6 & $-0,12$ & 165 & $-1,20$ & 26,67 & 0,52 & $P_{50}$ & 37,7 & 2,30 & $>P_{95}$ & 35,8 & 4,30 & $>P_{95}$ \\
\hline 18 & 79,7 & 0,74 & 178 & 0,37 & 25,15 & 0,77 & $P_{75}$ & 40,0 & 2,90 & $>P_{95}$ & 36,2 & 6,80 & $>P_{95}$ \\
\hline 19 & 74,3 & 0,04 & 164 & $-1,40$ & 27,62 & 0,82 & $P_{75}$ & 41,0 & 2,70 & $>P_{95}$ & 37,5 & 5,00 & $>P_{95}$ \\
\hline 20 & 78,0 & 0,57 & 174 & $-0,30$ & 25,76 & 1,00 & $P_{75}$ & 40,4 & 3,60 & $>P_{95}$ & 37,1 & 7,40 & $>P_{95}$ \\
\hline 21 & 85,0 & 1,27 & 172 & $-0,63$ & 28,73 & 2,26 & $P_{75}$ & - & & - & - & & - \\
\hline 22 & 74,0 & 0,17 & 165 & $-1,80$ & 27,18 & 1,62 & $P_{75}$ & 41,9 & 4,20 & $>P_{95}$ & 39,6 & 8,90 & $>P_{95}$ \\
\hline 23 & 82,0 & 0,97 & 173 & $-0,47$ & 27,40 & 1,70 & $P_{90}$ & 40,5 & 3,60 & $>P_{95}$ & 37,6 & 7,70 & $>P_{95}$ \\
\hline 24 & 72,0 & $-0,03$ & 170 & $-0,97$ & 24,91 & 0,67 & $P_{50}$ & - & & - & - & & - \\
\hline 25 & 79,5 & 0,72 & 168 & $-1,30$ & 28,17 & 2,00 & $P_{90}$ & 41,2 & 4,00 & $>P_{95}$ & 38,9 & 5,30 & $>P_{95}$ \\
\hline 26 & 87,0 & 1,47 & 168 & $-0,30$ & 30,82 & 3,10 & $P_{95}$ & - & & - & - & & - \\
\hline$-\mathrm{X} / \mathrm{DP}$ & $78,8 \pm 8,5$ & $0,56 \pm 0,82$ & $170,6 \pm 6,7$ & $-0,48 \pm 1,10$ & $27,0 \pm 1,9$ & $1,2 \pm 0,80$ & & $41,1 \pm 2,4$ & $3,6 \pm 1,00$ & & $38,5 \pm 2,5$ & $6,8 \pm 1,80$ & \\
\hline
\end{tabular}

$\mathrm{Z}=$ Valores de escore Z; IMC = Índice de Massa Corporal; $\mathrm{CB}=$ Circunferência Braquial; $\mathrm{CMB}=$ Circunferência Muscular do Braço 


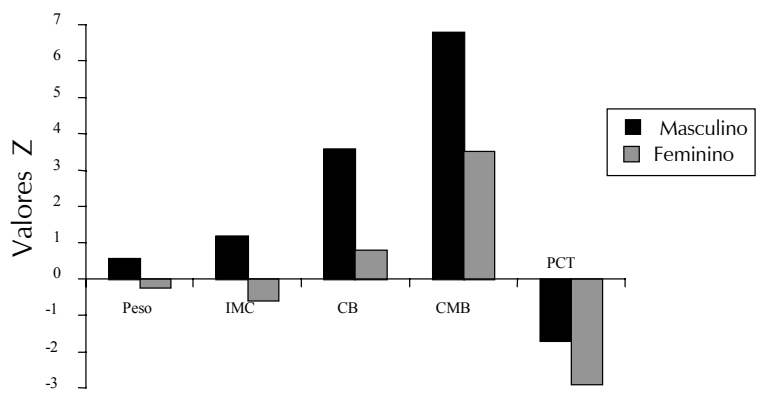

Figura 1. Escore $Z$ das variáveis antropométricas de atletas culturistas de ambos os sexos.

pélvicas, nádegas e coxas. O mínimo de adiposidade feminina é 12,0\% (gordura constitucional essencial), 4 vezes o valor do homem (3\%) (McArdle, 1991).

Atletas de culturismo são os que apresentam maior percentual de massa magra dentre todas as modalidades esportivas, atingindo cerca de 65,0\% do corpo, valor $20,0 \%$ acima dos sedentários (Spenst et al., 1993). A adiposidade corpórea de atletas de força (culturistas, levantadores de peso) atinge em média 5,0 a 15,7\% (Tabela $3)$. Dentre esses atletas o menor percentual lipídico (8,4\%) é o apresentado pelos culturistas (Fahey et al., 1975). Os resultados de adiposidade referidos na Tabela 3 são comparáveis a este estudo, onde no sexo masculino esta foi $6,9 \pm 1,5 \%$ e no feminino foi $8,1 \pm, 0,98 \%$ (Tabelas 4 e 5 ), sendo que os valores de referência citados por Petrosky (1995) e Pollock \& Jackson (1980), são de 4 a 9,0\% e de 8 a $12,0 \%$, para os sexos masculino e feminino, respectivamente.
O déficit de tecido adiposo observado atingiu $100,0 \%$ das atletas femininas e $15,0 \%$ dos atletas masculinos. Por esse indicador as mulheres seriam classificadas como desnutridas moderadas $(60,0 \%)$ ou graves $(40,0 \%)$ enquanto que apenas $11,5 \%$ dos atletas masculinos seriam considerados eutróficos, pois $73,0 \%$ eram desnutridos leves ou 15,5\% desnutridos moderados (Tabelas 2 e 5).

Deixando de usar o padrão local (Anselmo et al., 1992) como referência e empregando-se a distribuição percentual de autores de outros países (Frisancho, 1981; Cronk \& Roche, 1982), verifica-se situação semelhante ou seja 100,0\% dos homens estão com IMC acima da mediana $\left(P_{50}\right), 25,0 \%$ dos quais acima do $P_{90}$. Dentre as mulheres verificou-se $56,0 \%$ entre $P_{50}-P_{75}$ com as demais abaixo do $P_{25}$. A circunferência do braço e a cincunferência muscular do braço de todos os atletas situaram-se acima do $\mathrm{P}_{95}$ ao mesmo tempo que 92,3\% deles apresentam PCT menor que o $\mathrm{P}_{5}$. Os extremos mais representativos das mulheres foram $70,0 \%$ com CMB acima do $P_{95}$ e 90,0\% com PCT abaixo do $P_{5}$ (Tabelas 1, 2, 4 e 5). Desta forma, torna-se evidente a concordância entre os padrões de referência utilizados na discriminação dos atletas quanto aos desvios de adiposidade e massa muscular.

\section{CONCLUSÃO}

Estes resultados reiteram a inadequação do padrão antropométrico populacional para avaliação de atletas culturistas, sendo assim necessário o estabelecimento de padrões próprios ou ajustes (vias/fatores) aplicados aos padrões atualmente existentes.

Tabela 2. Peso, altura, circunferência braquial e medidas derivadas de atletas culturistas do sexo feminino.

\begin{tabular}{|c|c|c|c|c|c|c|c|c|c|c|c|c|c|}
\hline & \multicolumn{2}{|c|}{ Peso } & \multicolumn{2}{|c|}{ Altura } & \multicolumn{3}{|c|}{ IMC } & \multicolumn{3}{|c|}{$\mathrm{CB}$} & \multicolumn{3}{|c|}{$\mathrm{CMB}$} \\
\hline & $\mathrm{kg}$ & Z & $\mathrm{cm}$ & Z & $\mathrm{kg} / \mathrm{m}^{2}$ & z & Percentil & $\mathrm{cm}$ & Z & Percentil & $\mathrm{cm}$ & Z & Percentil \\
\hline 1 & 56,3 & $-0,62^{*}$ & 159 & 0,15 & 22,27 & $-0,72$ & $P_{50}$ & 32,8 & 0,71 & $P_{90}$ & 30,4 & 4,20 & $>P_{95}$ \\
\hline 2 & 55,4 & 0,11 & 161 & 0,28 & 21,37 & $-0,01$ & $P_{50}$ & 33,4 & 2,50 & $P_{90}$ & 31,0 & 5,60 & $>P_{95}$ \\
\hline 3 & 59,3 & $-0,30$ & 160 & 0,28 & 23,16 & $-0,50$ & $P_{50}$ & 33,6 & 1,00 & $P_{75}$ & 31,7 & 4,80 & $>P_{95}$ \\
\hline 4 & 50,4 & $-1,24$ & 153 & $-0,62$ & 21,53 & $-0,91$ & $P_{25}$ & 29,3 & 0,46 & $P_{50}$ & 27,0 & 2,50 & $P_{95}$ \\
\hline 5 & 48,7 & $-1,42$ & 160 & 0,28 & 19,02 & $-1,60$ & $P_{5}$ & 27,5 & $-0,92$ & $P_{25}$ & 25,0 & 1,50 & $P_{90}$ \\
\hline 6 & 46,5 & $-1,20$ & 157 & $-0,52$ & 18,86 & $-1,15$ & $P_{10}$ & 26,8 & $-0,21$ & $\mathrm{P}_{50}$ & 23,7 & 1,80 & $P_{90}$ \\
\hline 7 & 50,0 & $-0,70$ & 157 & $-0,52$ & 20,28 & $-0,51$ & $P_{25}$ & 28,1 & 0,63 & $\mathrm{P}_{50}$ & 24,7 & 2,30 & $\mathrm{P}_{90}$ \\
\hline 8 & 62,0 & 1,07 & - & & - & - & & 30,0 & 0,93 & $P_{75}$ & 27,1 & 3,60 & $>P_{95}$ \\
\hline 9 & 54,3 & $-0,04$ & 159 & $-0,12$ & 21,48 & 0,04 & $\mathrm{P}_{50}$ & 30,5 & 1,30 & $P_{75}$ & 28,2 & 4,20 & $>P_{95}$ \\
\hline 10 & 67,0 & 1,80 & 178 & 3,70 & 21,15 & $-0,11$ & $P_{50}$ & 31,7 & 1,60 & $P_{75}$ & 28,7 & 4,40 & $>P_{95}$ \\
\hline${ }^{x} / D P$ & $55 \pm 6,4$ & $-0,25 \pm 1,00$ & $160,4 \pm 7,0$ & $0,32 \pm 1,30$ & $21 \pm 1,4$ & $-0,6 \pm 0,50$ & & $30,4 \pm 2,5$ & $0,8 \pm 0,90$ & & $27,8 \pm 2,7$ & $3,5 \pm 1,40$ & \\
\hline
\end{tabular}

Z = Valores de escore Z; IMC = Índice de Massa Corporal; CB = Circunferência Braquial; CMB = Circunferência Muscular do Braço 
Tabela 3. Adiposidade (\%) e Índice de Massa Corpórea (IMC) de atletas culturistas ou levantadores de peso encontrados na literatura.

\begin{tabular}{|c|c|c|c|c|c|}
\hline \multicolumn{3}{|c|}{$\%$ Gordura } & \multicolumn{2}{|c|}{$\mathrm{IMC}\left(\mathrm{kg} / \mathrm{m}^{2}\right)$} & \multirow{2}{*}{ Autores } \\
\hline & Homem & Mulher & Homem & Mulher & \\
\hline 5,0 & $\pm 2,0$ & $9,0 \pm 1,0$ & $27,4^{*}$ & $20,7^{*}$ & Kleiner et al. (1994) \\
\hline 5,8 & $\pm 2,0$ & - & $22,6 \pm 0,6$ & - & Mourier et al. (1997) \\
\hline 7,2 & $\pm \quad 1,6$ & $8,4 \pm 1,4$ & $26,3^{*}$ & $19,8^{*}$ & Sandoval et al. (1989) \\
\hline 9,6 & $\pm 1,7$ & - & - & - & Tarnopolsky et al. (1988) \\
\hline 10,6 & $\pm \quad 3,2$ & - & $26,6 \pm 2,7$ & - & Porto (1999) \\
\hline 11,1 & $\pm \quad 4,0$ & - & $24,6^{*}$ & - & Tarnopolsky et al. (1991) \\
\hline 12,0 & $\pm 5,4$ & $17,4 \pm 3,8$ & $23,6^{*}$ & $20,9^{*}$ & Pavlou et al. (1993) \\
\hline 13,6 & $\pm 1,5$ & $14,4 \pm 3,8$ & $25,4^{*}$ & $21,2^{*}$ & Crist et al. (1988) \\
\hline 15,1 & $\pm \quad 5,2$ & - & $28,2 \pm 3,3$ & - & Maestá (1999) \\
\hline 15,7 & $\pm \quad 3,2$ & - & - & - & Dragan et al. (1985) \\
\hline
\end{tabular}

(Cálculo realizado a partir do peso e da altura que os autores forneceram no trabalho

Tabela 4. Dados antropométricos de adiposidade de atletas culturistas do sexo masculino.

\begin{tabular}{|c|c|c|c|c|c|c|c|}
\hline & \multicolumn{3}{|c|}{ PCT } & \multirow{2}{*}{$\begin{array}{l}\mathrm{PCP} \\
\mathrm{mm}\end{array}$} & \multirow{2}{*}{$\begin{array}{c}\text { PCSE } \\
\mathrm{mm}\end{array}$} & \multirow{2}{*}{$\begin{array}{r}\text { PCSI } \\
\mathrm{mm}\end{array}$} & \multirow{2}{*}{$\begin{array}{c}\text { Gordura } \\
\%\end{array}$} \\
\hline & $\mathrm{mm}$ & Z & Percentil & & & & \\
\hline 1 & 2,6 & 0,09 & $<\mathrm{P}_{5}$ & 2,2 & 8,7 & 3,2 & 9,7 \\
\hline 2 & 3,4 & $-1,70$ & $<\mathrm{P}_{5}$ & 2,9 & 8,1 & 3,1 & 6,1 \\
\hline 3 & 2,4 & $-2,00$ & $<\mathrm{P}_{5}$ & 2,3 & 7,5 & 2,8 & 8,3 \\
\hline 4 & 3,6 & $-1,70$ & $<\mathrm{P}_{5}$ & 2,7 & 5,2 & 3,1 & 6,7 \\
\hline 5 & 2,3 & $-2,06$ & $<\mathrm{P}_{5}$ & 1,8 & 5,8 & 2,3 & 8,4 \\
\hline 6 & 3,5 & $-1,70$ & $<\mathrm{P}_{5}$ & 3,1 & 8,6 & 3,1 & 6,3 \\
\hline 7 & 2,8 & $-1,80$ & $<\mathrm{P}_{5}$ & 1,9 & 7,2 & 3,1 & 6,5 \\
\hline 8 & 2,2 & $-1,90$ & $<\mathrm{P}_{5}$ & 2,2 & 6,7 & 2,8 & 4,8 \\
\hline 9 & 3,0 & $-1,90$ & $<P_{5}$ & 2,1 & 7,7 & 2,3 & 7,1 \\
\hline 10 & 2,2 & $-0,31$ & $<\mathrm{P}_{5}$ & 1,6 & 7,6 & 2,1 & 6,7 \\
\hline 11 & 7,0 & $-1,20$ & $\mathrm{P}_{25}$ & 6,8 & 8,1 & 3,4 & 8,3 \\
\hline 12 & 2,0 & $-1,90$ & $<\mathrm{P}_{5}$ & 2,7 & 6,6 & 3,1 & 6,1 \\
\hline 13 & 2,5 & $-1,80$ & $<\mathrm{P}_{5}$ & 1,5 & 8,5 & 3,1 & 5,8 \\
\hline 14 & 1,4 & $-2,00$ & $<\mathrm{P}_{5}$ & 1,3 & 8,7 & 2,6 & 6,2 \\
\hline 15 & 2,1 & $-2,10$ & $<\mathrm{P}_{5}$ & 3,8 & 6,2 & 3,7 & 7,8 \\
\hline 16 & 2,4 & $-2,04$ & $<\mathrm{P}_{5}$ & 2,2 & 7,7 & 2,4 & 7,3 \\
\hline 17 & 3,6 & 1,80 & $<\mathrm{P}_{5}$ & 6,3 & 8,9 & 5,4 & 11,8 \\
\hline 18 & 2,6 & $-1,80$ & $<\mathrm{P}_{5}$ & 3,0 & 4,6 & 2,5 & 4,4 \\
\hline 19 & 2,2 & $-2,08$ & $<\mathrm{P}_{5}$ & 1,9 & 5,8 & 2,3 & 6,5 \\
\hline 20 & 5,2 & $-1,45$ & $P_{10}$ & 6,0 & 7,1 & 3,2 & 7,3 \\
\hline 21 & 2,9 & $-1,80$ & $P_{5}$ & 2,7 & 7,5 & 3,2 & 6,9 \\
\hline 22 & 2,4 & $-1,80$ & $P_{5}$ & 2,8 & 10,1 & 2,8 & 6,9 \\
\hline 23 & 3,4 & $-1,70$ & $P_{5}$ & 4,5 & 7,1 & 3,8 & 6,4 \\
\hline 24 & 2,6 & $-1,80$ & $P_{5}$ & 1,7 & 7,7 & 2,6 & 5,8 \\
\hline 25 & 2,8 & $-1,80$ & $P_{5}$ & 3,5 & 7,5 & 3,6 & 6,4 \\
\hline 26 & 2,8 & $-1,80$ & $P_{5}$ & 4,9 & 7,2 & 4,4 & 6,9 \\
\hline$\overline{\mathrm{X}} / \mathrm{DP}$ & $2,9 \pm 1,1$ & $-1,7 \pm 0,5$ & & $3,0 \pm 1,5$ & $7,4 \pm 1,2$ & $3,1 \pm 0,7$ & $6,9 \pm 1,5$ \\
\hline
\end{tabular}

$Z=$ Valores de escore Z; PCT = Prega Cutânea Tricipital; PCP = Prega Cutânea da Panturrilha; PCSE = Prega Cutânea Subescapular, PCSI = Prega Cutânea Suprailíaca. 
Tabela 5. Dados antropométricos de adiposidade de atletas culturistas do sexo feminino.

\begin{tabular}{|c|c|c|c|c|c|c|c|}
\hline & \multicolumn{3}{|c|}{ РCT } & \multirow{2}{*}{$\frac{\text { PCAb }}{\mathrm{mm}}$} & \multirow{2}{*}{$\frac{\mathrm{PCCx}}{\mathrm{mm}}$} & \multirow{2}{*}{$\frac{\text { PCSI }}{\mathrm{mm}}$} & \multirow{2}{*}{$\begin{array}{c}\text { Gordura } \\
\%\end{array}$} \\
\hline & $\mathrm{mm}$ & Z & Percentil & & & & \\
\hline 1 & 5,2 & $-2,52$ & $<\mathrm{P}_{5}$ & 4,5 & 8,0 & 2,5 & 8,2 \\
\hline 2 & 4,0 & $-3,42$ & $<\mathrm{P}_{5}$ & 4,4 & 9,6 & 3,0 & 8,3 \\
\hline 3 & 4,1 & $-2,70$ & $<\mathrm{P}_{5}$ & 3,6 & 7,2 & 1,9 & 7,4 \\
\hline 4 & 3,7 & $-2,70$ & $<\mathrm{P}_{5}$ & 4,2 & 5,0 & 2,8 & 7,1 \\
\hline 5 & 5,3 & $-2,50$ & $<\mathrm{P}_{5}$ & 9,5 & 7,0 & 5,6 & 10,3 \\
\hline 6 & 6,6 & $-2,80$ & $<\mathrm{P}_{5}$ & 2,6 & 3,5 & 7,4 & 7,9 \\
\hline 7 & 10,0 & $-2,10$ & $P_{5}$ & 4,5 & 5,5 & 3,9 & 9,0 \\
\hline 8 & 4,6 & $-3,30$ & $<\mathrm{P}_{5}$ & 4,7 & 8,5 & 3,7 & 8,3 \\
\hline 9 & 4,2 & $-3,40$ & $<\mathrm{P}_{5}$ & 6,0 & 2,5 & 5,2 & 7,3 \\
\hline 10 & 4,0 & $-3,40$ & $<\mathrm{P}_{5}$ & 3,1 & 7,9 & 2,5 & 7,3 \\
\hline$\overline{\mathrm{X}} / \mathrm{DP}$ & $5,2 \pm 1,9$ & $-2,9 \pm 0,5$ & & $4,7 \pm 1,9$ & $6,47 \pm 2,3$ & $3,85 \pm 1,7$ & $8,1 \pm 0,98$ \\
\hline
\end{tabular}

$\mathrm{Z}=$ Valores de escore Z; PCT = Prega Cutânea Tricipital; PCAb = Prega cutânea Abdominal; PCCX = Prega Cutânea da coxa; PCSI = Prega Cutânea Suprailíaca

A implicação prática desses contrastes vai desde a classificação equivocada desses atletas como desnutridos (pelo critério adiposidade) ou sobrepeso ou ainda obesos (pelo critério massa magra) até erros no cálculo da adequação alimentar por unidade de peso ou, mais apropriadamente, por peso de massa magra.

\section{REFERÊNCIAS BIBLIOGRÁFICAS}

ANSELMO M.A.C., BURINI, R.C., ANGELELI, A.Y.O., MOTA, N.G.S., CAMPANA, A.O. Avaliação do estado nutricional de indivíduos adultos sadios de classe média. Ingestão energética e protéica, antropometria, exames bioquímicos do sangue e teste de imunocompetência. Revista de Saúde Pública, São Paulo, v.26, n.1, p.46-53, 1992.

CRIST, D.M., PEAKE, G.T., EGAN, P.A., WATERS, D.L. Body composition response to exogenous $\mathrm{GH}$ during training in highly conditioned adults. Journal of Applied Physiology, Bethesda, v.65, n.2, p.579-584, 1988.

CRONK, C.E., ROCHE, A.F. Race and sex specific reference data for triceps and subscapular skinfolds and weight/stature ${ }^{2}$. American Journal of Clinical Nutrition, Bethesda, v.35, n.2, p.347-357, 1982.

DRAGAN, G.I., VASILIU, A., GEORGESCU, E. Researches concerning the effects of refit on elite weightlifters. Journal of Sports Medicine and Physical Fitness, Torino, v.25, n.4, p.246-250, 1985.

FAHEY T.D., AKKA, L., ROL, R.D. Body composition and $\mathrm{VO}_{2 \max }$ of exceptional weight-trained athletes. Journal of Applied Physiology, Bethesda, v.39, n.4, p.559-561, 1975.

FLECK, S.J., KRAEMER W.J. Resistance Training Sports. In:FLECK, S.J., KRAEMER, W.J. Designing resistance training programs. 2.ed. Champaing IL : Human Kinetics, 1997. p. 231-244.
FRISANCHO, A.R. New norms of upper limb fat and muscle areas for assessment of nutritional status. American Journal of Clinical Nutrition, Bethesda, v.34, n.11, p.2540-2545, 1981.

KLEINER, S.M., BAZZARRE, T.L., AINSWORTH, B.E. Nutritional status of nationally ranked elite bodybuilders. International Journal of Sport Nutrition, Champaign IL, v.4, n.1, p.54-69, 1994.

MAESTÁ, N. Efeito da oferta nitrogenada sobre a composição corpórea e turnover protéico total de culturistas em treinamento. Botucatu, 1999. 82p. Dissertação (Mestrado em Fisiopatologia em Clínica Médica - Metabolismo e Nutrição) - Faculdade de Medicina, Universidade Estadual Paulista, 1999.

McARDLE, W.D. Avaliação da composição corporal. In: MCARDLE, W.D., KATCH, F.I., KATCH, V.L. Fisiologia do exercício: energia, nutrição e desempenho humano. 3.ed. Rio de Janeiro : Guanabara, 1991. p.387-409.

MOURIER, A., BIGARO, A.X., KERVILER, E., ROGER, B., LEGRAND, H., GUEZENNEC, C.Y. Combined effects of caloric restriction and branched chain supplementation on body composition and exercise performance in elite wrestlers. International Journal of Sport Medicine, Champaign IL, v.18, n.1, p.47-55, 1997.

PAVLOU, K.N. Energy needs of the elite athlete. World Review of Nutrition and Dietetics, Basel, v.71, p.9-20, 1993. (Volume Único).

PETROSKY, E.L. Desenvolvimento e validação de equações generalizadas para a estimativa da densidade corporal em adultos. Santa Maria, 1995. p.1-124. Tese (Doutorado em Educação Física) - Universidade Federal de Santa Maria, 1995.

POLLOCK, M.L., JACKSON, A.S. Measurement of cardiorespiratory fitness and body composition in the clinical setting. Comprehensive Therapy, Ayer MA, v.6, n.9, p.12-27, 1980. 
PORTO, M. Modulação dietética (protéico/glicídica) do ganho de massa, força e índice de fadiga muscular em atletas culturistas em treinamento. Rio Claro, 1999, 85p. Dissertação (Mestrado em Ciências da Motricidade - Motricidade Humana) - Instituto de Biociências, Universidade Estadual Paulista, 1999

SANDOVAL, W.M., HEYWARD, V.H., LYONS, T.M. Comparison of body composition, exercise and nutritional profiles of female and male bodybuilders at competition. Journal of Sports Medicine and Physical Fitness, Torino, v.29, n.1, p.63-70, 1989.

SAWAYA, A.L. Desnutrição energético protéica. In: SAWAYA A.L., FERRARI A.A. Desnutrição urbana no Brasil em um período de transição. São Paulo : Cortez. 1997. p.19-33.

SIRI, M.E. Body composition from fluid spaces and density. In: BROZEK J., HENSCHEL, A. Techniques for measuring body composition. Washington DC : National Academy of Science, 1961. p.223-244
SPENST, L.F., MARTIN, A.D., DRINKWATER, D.T. Muscle mass of competitive male athletes. Journal Sports Sciences, London, v.11, n.1, p.3-8, 1993.

TARNOPOLSKY, M., MacDOUGALL, J.D., ATKINSON, S. Influence of protein intake and training status on nitrogen balance and lean body mass. Journal Applied Physiology, Bethesda, v.64, n.1, p.187-193, 1988.

TARNOPOLSKY, M.A., ATKINSON, S.A., MacDOUGALL, J.D., SENOR, B.B., LEMON, P.W.R., SCHWARCZ, H. Whole body leucine metabolism during and after resistance exercise in fed humans. Medicine and Science in Sports and Exercise, Madison, v.23, n.3, p.326-333, 1991.

WORLD HEALTH ORGANIZATION. Physical status: the use and interpretation of anthropometry. Geneva, 1995. p.1-452. (Who Report Series, 854).

Recebido para publicação em 25 de maio e aceito em 7 de outubro de 1999. 